\title{
Online algorithm for possibilitic fuzzy clustering based on evolutionary cat swarm optimization
}

\author{
A. Shafronenko, Ye. Bodyanskiy \\ Kharkiv National University of Radio Electronics \\ Corresponding author. E-mail: alina.shafronenko@nure.ua, yevgeniy.bodyanskiy@nure.ua
}

Paper received 21.01.19; Accepted for publication 28.01.19.

\section{https://doi.org/10.31174/SEND-NT2019-193VII23-22}

Abstract. The problem of clustering of multidimensional observations is often found in many applications related to data mining and exploratory data analysis. The traditional approach to solving these problems requires that every observation could belong to only one cluster at a more natural is situations when a feature vector with the various possible levels of memberships can belong to multiple classes. This situation is the subject of fuzzy cluster analysis, rapidly developing now. We propose online adaptive approach for this task solving.

Keywords: Fuzzy clustering, learning rule, cat swarm optimization, tracing mode, seeking mode.

Introduction. At present, methods of computational intelligence are widely used to solve many complex problems. One of the main areas of computational intelligence are evolutionary algorithms that essentially represent certain mathematical models of biological organisms evolutions. The dataset problem described by vector image clustering often occurs in many data mining applications, but recently the focus has been on fuzzy clustering $[1,2,3]$ when processing vector images with different levels of probability, possibility or membership may belong to more than one class. Kohonen self-organizing maps [4] are a very effective means of coordinated online clustering, the use of which permits to solve the task in online mode and the evolutionary algorithms that permit to improve data clusterization in case, when the data is processed sequentially in online mode.

Problem statement. The problem of fuzzy clustering of data arrays is considered in the conditions when the formed clusters arbitrarily overlap in the space of features. The source information for solving the problem is an array of multidimensional data vectors, formed by a sample of observations $X=(x(1), x(2), \ldots, x(k), \ldots x(N)) \subset R^{n}$ where $k$ in the general case the observation number in the initial array, $\quad x(k)=\left(x_{1}(k), \ldots, x_{i}(k), \ldots x_{n}(n)\right)^{T}$. The result of clustering is the partition of this array on m disjoint classes $C l_{j}$ with prototype-centroids $C l_{j} \in R^{n}, j=1,2, \ldots, m$, and computing of membership levels $0 \leq U_{j}(k) \leq 1$ of each observation $x(k)$ to every cluster $C l_{j}$.

Adaptive algorithm for possibilistic fuzzy clustering. The main drawback of the classic probabilistic clustering algorithms [1-3] associated with restrictions on membership levels, the sum of which has to be equal to unity. This situation led to the creation of possiblistic fuzzy clustering algorithms [5].

The goal function of possibilistic clustering has the form

$$
E\left(U_{j}(k), c_{j}, \mu_{j}\right)=\sum_{k=1}^{N} \sum_{j=1}^{m} U_{j}^{\beta}(k) D^{2}\left(\tilde{x}_{k}, c_{j}\right)+\sum_{j=1}^{m} \mu_{j} \sum_{k=1}^{N}\left(1-U_{j}(k)\right)^{\beta}
$$

where $\mu \geq 0$ - the scalar parameter thatspecifies the distance at which the level of membership equals 0.5 , i.e. if $D^{2}\left(\tilde{x}_{k}, c_{j}\right)=\mu_{j}$, then $c_{j}(k)=0.5$.

Minimizing the equation (1) accordingly, $U_{j}(k), c_{q}$ and $\mu_{j}$ we obtain system of equations

$$
\left\{\begin{array}{c}
U_{j}^{(\tau+1)}(k)=\frac{1}{1+\left(\frac{D^{2}\left(\tilde{x}(k), c_{j}^{(\tau)}\right)}{\mu_{j}^{(\tau)}}\right)^{\frac{1}{\beta-1}}}, \\
c_{j}^{(\tau+1)}=\frac{\sum_{k=1}^{N}\left(U_{j}^{(\tau+1)}(k)\right)^{\tilde{x}}(k)}{\sum_{k=1}^{N}\left(U_{j}^{(\tau+1)}(k)\right)^{\beta}}, \\
\mu_{j}^{(\tau+1)}=\frac{\sum_{k=1}^{N}\left(U_{j}^{(\tau+1)}(k)\right)^{\beta} D^{2}\left(\tilde{x}(k), c_{j}^{(\tau+1)}\right)}{\sum_{k=1}^{N}\left(U_{j}^{(\tau+1)}(k)\right)^{\beta}},
\end{array}\right.
$$

in case when the $\beta=2$ we come to a decision, that has the form (analogue of classic FCM [1]):

$$
\left\{\begin{array}{l}
U_{j}^{(\tau+1)}(k)=\frac{1}{1+\frac{\left\|\tilde{x}(k)-c_{j}^{(\tau)}\right\|^{2}}{\mu_{j}^{(\tau)}}}, \\
c_{j}^{(\tau+1)}=\frac{\sum_{k=1}^{N}\left(U_{j}^{(\tau)}(k)\right)^{2} \tilde{x}(k)}{\sum_{k=1}^{N}\left(U_{j}^{(\tau)}(k)\right)^{2}}, \\
\mu_{j}^{(\tau+1)}=\frac{\sum_{k=1}^{N}\left(U_{j}^{(\tau)}(k)\right)^{2}\left\|\tilde{x}(k)-c_{j}^{(\tau+1)}\right\|^{2}}{\sum_{k=1}^{N}\left(U_{j}^{(\tau)}(k)\right)^{2}} .
\end{array}\right.
$$

In the online mode the formulas (2), (3) can be written as $[6,7]$ 


$$
\begin{aligned}
& \left\{\begin{array}{l}
U_{j}(k+1)=\frac{1}{1+\left(\frac{D^{2}\left(\tilde{x}(k+1), c_{j}(k)\right)}{\mu_{j}(k)}\right)^{\frac{1}{\beta-1}}}, \\
c_{j}(k+1)=c_{j}(k)+\eta(k+1) U_{j}^{\beta}(k+1)\left(\tilde{x}(k+1)-c_{j}(k)\right), \\
\mu_{j}(k+1)=\frac{\sum_{p=1}^{k+1} U_{j}^{\beta}(p) D^{2}\left(\tilde{x}(p), c_{j}(k+1)\right)}{\sum_{p=1}^{k+1} U_{j}^{\beta}(p)} \\
\text { and } \\
U_{j}(k+1)=\frac{1}{1+\frac{\left\|\tilde{x}(k)-c_{j}(k)\right\|^{2}}{\mu_{j}(k)}} \\
c_{j}(k+1)=c_{j}(k)+\eta(k+1) U_{j}^{2}(k+1)\left(\tilde{x}(k+1)-c_{j}(k)\right), \\
\mu_{j}(k+1)=\frac{\sum_{p=1}^{k+1} U_{j}^{2}(p)\left\|\tilde{x}(p)-c_{j}(k+1)\right\|^{2}}{\sum_{p=1}^{k} U_{j}^{2}(p)}
\end{array}\right.
\end{aligned}
$$
mode.

that permits to solve the fuzzy clustering task in online

$$
\tilde{n}_{p}(\tau+1)=\tilde{n}_{p}(\tau)-\alpha\left(\tilde{n}_{p}(\tau)-\tilde{n}_{p}(\tau-1)\right)-\eta \hat{\nabla} E_{M}\left(c_{p}(\tau)\right)+\eta \xi \Xi(\tau)
$$

Here $\tilde{n}_{p}(\tau+1)$ - state of $p$-th cat of swarm on $\tau$-th iteration of the search, $\alpha$ - parameter that determines the inertia properties of the tracing mode. Thus, when $\alpha=0$, process optimization approaches to the standard gradient search, i.e. the seeking mode in the swarm, with $0<\alpha<1$ the optimization process acquires the inertial properties of a "heavy ball" type, but it may not fade in the vicinity of a deep extremum, $\eta$ - seeking mode step, $\hat{\nabla} E\left(c_{p}(\tau)\right)$ gradient estimate of the goal function (1) in the neighborhood of the point $\tilde{n}_{p}(\tau), \Xi(\tau)$ - a random component that introduces additional stochastic motions into the tracing process, $\eta_{\xi}$ - parameter that specifies the amplitude of these movements.

Thus, each cat can simultaneously be in seeking and tracing modes and with a sufficient number of cats in a swarm, provides a search for a global extremum.
To find the global extremum of (1), it is expedient to use the so-called bio-inspired evolutionary particles swarm optimization algorithms [8], that are being developed and explored at this time in the framework of the general theory and practice of computational intelligence. Among the swarm algorithms, one of the fastest ones are the so-called algorithms of the cats swarm $[9,10]$, that proved to be effective in solving a wide range of Data Mining tasks.

As part of this approach, a cat swarm optimization assumes that each cat of the swarm can be in one of two states: the seeking mode and the tracing mode. In this case, the seeking mode is associated with slow motions with a small amplitude around the initial position (scanning space in the vicinity of the current position), and the tracing mode that is determined by fast jumps with high amplitude and allows the to leave each particular cat from the local extremum if it is there. The combination of local scanning and rapid changes in the current state allows to increase the likelihood of finding a global extremum in comparison with the traditional methods of multyextremal optimization.

In the general case, both of these modes for each of the cats swarms can be described by the recurrent optimization procedure
Experimental research. Online algorithm for possibilitic fuzzy clustering based on evolutionary cat swarm optimization (OPCSO) were performed on 2 data samples. Source data clustering using the Fuzzy C-Means, Adaptive possibilistic fuzzy method data clustering, Gustafson-Kessel and APCSO. The simulation parameters are presented in Table1. Result of clusterization are presented in Table 2.

Table 1 - Parameters of cat swarm optimization algorithm (CSO)

\begin{tabular}{|l|l|}
\hline Parameters & Value \\
\hline SRD & Random $[0,1]$ \\
\hline Seeking memory Pool (SMP) & 5 \\
\hline Population size & Number of clusters \\
\hline $\mathrm{r}_{1}$ & Random in $[0,1]$ \\
\hline $\mathrm{c}_{1}$ & Const \\
\hline SPC & Random in $[0,1]$ \\
\hline Number of iteration & Manually \\
\hline
\end{tabular}

Table 2 - Evaluation of the quality of fuzzy clustering methods data

\begin{tabular}{|l|c|c|c|}
\hline Data clustering methods & PC & SC & XB \\
\hline Fuzzy C-Means & 0.50 & 1.62 & 0.19 \\
\hline Gustafson-Kessel & 0.27 & 1.66 & 1.62 \\
\hline Adaptive fuzzy possibilistic data clustering & 0.26 & 1.22 & $\mathbf{0 . 0 1}$ \\
\hline $\begin{array}{l}\text { Online algorithm for possibilitic fuzzy clustering based on } \\
\text { evolutionary cat swarm optimization (OPCSO) }\end{array}$ & $\mathbf{0 . 2 4}$ & $\mathbf{0 . 6 9}$ & 0.15 \\
\hline
\end{tabular}

Also conducted a comparative analysis of the quality of clustering data on the main characteristics quality ratings, such as: Partition Coefficient (PC), Partition Index (SC), Xie and Beni's Index (XB) of existing clustering methods and proposed method. As can be seen by the results of experiments, the proposed algorithm shows quite good results clustering quality.
Conclusion. The proposed approach is quite simple in numerical implementation, has high performance and provides high quality of fuzzy clustering of large arrays. It is shown that it can be solved on the basis of Kohonen's selforganizing neuro-fuzzy network "Winner Takes More" recurrent algorithms for fuzzy clustering and cat swarm optimization algorithms. 


\section{REFERENCES}

1. J.C. Bezdek. Pattern Recognition with Fuzzy Objective Function Algorithms. Plenum Press, New York, 1981.

2. F Hoeppner, F. Klawonn, R. Kruse, T. Runker. Fuzzy Clustering Analysis: Methods for Classification, Data Analysis and Image Recognition. Chichester, John Wiley \&Sons, 1999.

3. R. Xu, D.C. Wunsch. Clustering. Hoboken, N.J. John Wiley \& Sons, Inc., 2009.

4. T. Kohonen. Self-Organizing Maps. Berlin: Springer-Verlag, 1995.

5. R. Krishnapuram, J.M. Keller. A possibilistic approach to clustering. Fuzzy Systems, 1993, 1, №2, P.98-110.

6. Ye. Bodyanskiy. Computational intelligence techniques for data analysis. Lecture Notes in Informatics. Bonn: GI, 2005, V. P-72, P. 15-36.
7. Ye. Gorshkov, V. Kolodyazhniy, Ye. Bodyanskiy. New recursive learning algorithms for fuzzy Kohonen clustering network. Proc. 17th Int. Workshop on Nonlinear Dynamics of Electronic Systems. (Rapperswil, Switzerland, June 21-24, 2009) Rapperswil, Switzerland, 2009, P. 58-61.

8. Grosan C., Abraham A., Chis M. Swarm intelligence in Data Mining - Studies in Computational Intelligence. - 2006. - 34. - P. $1-20$.

9. Chu S.-C., Tsai P.-W., Pan J.S. Cat swarm optimization // Lecture Notes in Artificial Intelligence. - 4099. - Berlin Heidelberg: Springer-Verlag, 2006. - P. 854-858.

10. Chu S.-C., Tsai P.-W. Computational Intelligence based on the behavior of cats // Int. J. of Innovative Computing, Information, and Control. - 2007. - 3. - №1. - P.163 - 173. 